\title{
ANNOTATIONS
}

\section{A Higher Diploma in Ophthalmology}

The recent establishment of the Diploma in Ophthalmic Medicine and Surgery by the Conjoint Board of the Royal College of Physicians and Surgeons does not meet the need for a higher diploma in Ophthalmology. From one point of view, indeed, it accentuates the need ; for the D.O.M. and S. is of the nature of a "pass" qualification, and if it is allowed to stand alone may come to be regarded as a criterion of a higher standard of knowledge and efficiency than it really represents. What is still required is a higher grade examination which will give reliable evidence that a successful candidate is well equipped to hold the post of ophthalmic surgeon at a general hospital. The usual criterion for ophthalmic surgeons to a hospital is the possession of the F.R.C.S.Eng., but this gives no evidence of special qualification in ophthalmology. In addition to the ordinary requirements of a registrable medical qualification, the candidate for the post of ophthalmic surgeon should have (1) such knowledge of the principles of surgery and pathology as is demanded of the general surgeon; (2) a special knowledge of the anatomy aud physiology of the eye and of those structures which are in physiological and surgical relation to the eye; (3) a sound knowledge of the optics of the eye and of those instruments which are employed for the diagnosis and treatment of ocular disorders; (4) a special knowledge of ophthalmic surgery and medicine.

It will be noted that a high level of knowledge of general surgery and pathology is put at the head of the requirements and it would be lamentable if any concession were made upon this point. The standard hitherto has been that of the F.R.C.S.Eng., and such it should remain. At the same time it must be freely admitted that it is illogical and unnecessary to demand an extensive knowledge of the regional surgery of other parts of the body from a man who is definitely embarked upon a career as an ophthalmic surgeon. His time can be much better occupied in other directions.

The other requirements mentioned will be universally admitted, but there may be some difference of opinion as to the relative rôles to be allotted to surgery and medicine. In the past ophthalmology has generally been regarded as essentially surgical, and so far as hospital and private practice is concerned this view is justified. At the same time it is imperative that the medical aspect should receive adequate attention.

It is eminently desirable that the traditions which have hitherto held sway in England should be maintained. It follows that the higher diploma in ophthalmology should be granted either by the 
Royal College of Surgeons or by the Conjoint Board of the Royal College of Physicians and Royal College of Surgeons. The first suggestion is more in keeping with tradition than the second, and it would not be a difficult task to devise a modification of the fellowship examination which would meet all the requirements. If such an F.R.C.S. in Ophthalmology were instituted it would retain all the high reputation of the present F.R.C.S., and would soon become the recognized qualification for the ophthalmic post on a hospital staff.

\section{The Pennsylvania University Graduate School of Ophthalmology}

ON October 1st of this year the University of Pennsylvania, which gave a preliminary course in Ophthalmology in 1919, entered upon its first year of the full course of instruction. The Graduate School of Medicine is the outcome of the taking over by the University of Pennsylvania of the Medico-Chirurgical College and the Philadelphia Polyclinic, the latter institution having been for many years devoted to graduate teaching.

It will be seen that these recommendations are in complete accordance with those made by the British Council of Ophthalmologists. (B.J.O., December, 1919, p. 558.)

Two courses are offered. First, a ten months' course, eight months of which are devoted to graduated systematic teaching by laboratory work, didactic and clinical lectures, demonstrations, clinical work, operative clinics and conferences, and two months devoted to purely clinical work. At the end of the course examinations will be held, and the successful candidates will be given certificates of attendance, or will be permitted to enter upon a second year of work as candidates for a degree.

The second year is devoted to advanced studies, practical clinical work as clinical assistants to the departmental chiefs, and to research work.

The classes are limited to sixteen students and are sectioned so as to admit of personal supervision and instruction by each of the chiefs of the departments.

The Ophthalmological Staff consists of Dr. George Crampton, in charge of the Physiologic Optics and Refraction; Dr. L. Webster Fox, Surgical Clinics; Dr. Thomas B. Holloway, External Diseases and Neuro-Ophthalmology; Dr. Luther C. Peter, Ocular Muscles and Perimetry; Dr. William T. Shoemaker, Surgery of the Eye ; and Dr. William Zentmayer, Ophthalmoscopy and Medical Ophthalmology. The Department of Pathology and Bacteriology is under the supervision of Dr. John Kolmer. 